\title{
Comparison of nonlinear Von Karman and Cosserat theories in very large deformation of skew plates
}

\author{
Aazam Ghassemi ${ }^{1}\left[\right.$. Mohsen Hassani ${ }^{1} \cdot$ Soheil Oveissi $^{1}$
}

Received: 21 July 2017 / Accepted: 24 January 2018 / Published online: 10 February 2018

(c) The Author(s) 2018. This article is an open access publication

\begin{abstract}
Prediction of plate behavior in large deformation is one of the important problems in plate theories. Cosserat theory is an advanced theory for simulation of plates in very large deformation, but it is complex from mathematical viewpoint. Another theory that has been used extensively for large deformation problems is nonlinear Von Karman theory which is easy for formulation and computation. In this paper, these theories were compared for rectangular and skew plates in simply supported and clamped boundary conditions to propose the acceptable range of using nonlinear Von Karman in very large deformation as a simpler theory. Higher order shear deformation plate theory was used with Von Karman nonlinearity. Whole domain method was employed for numerical solution. Each theory was validated with the literature for verification of the numerical method. Deflection and stress distribution were compared from small to very large deformations. The obtained results show that two theories were matched up to the maximum nondimensional deflection of 5 and 3 for simply supported and clamped boundary conditions, respectively. Moreover, by increasing the skew angle, the consistency of two theories would decrease even in deflections smaller than the thickness of the plate.
\end{abstract}

Keywords Cosserat theory · Von Karman nonlinearity $\cdot$ Large deformation $\cdot$ Higher order shear deformation plate theory Skew plate

\section{List of symbols}

$h \quad$ Thickness of the plate

$u_{01} \quad$ Midplane displacements in $x_{1}$ direction

$u_{02} \quad$ Midplane displacements in $x_{2}$ direction

$u_{03} \quad$ Displacement of plate in $x_{3}$ direction

$\Psi_{0 x_{1}} \quad$ Midplane rotation of the normal around the $x_{1}$

$\Psi_{0 x_{2}} \quad$ Midplane rotation of the normal around the $x_{2}$

$N_{i} \quad$ Membrane

$Q_{i} \quad$ Transverse shear force

$M_{i} \quad$ Bending moment per unit length

$P_{i} \quad$ Higher order bending moment

$R_{i} \quad$ Higher order shear force

$\delta U \quad$ Virtual strain energy

$\delta V \quad$ Virtual work

$\mathbf{s} \quad$ Shape function matrix

$\widehat{\mathbf{x}} \quad$ The general coordinate system

0 Superscript, before deformation

$t \quad$ Superscript, after deformation

Aazam Ghassemi

aazam77@yahoo.com; a_ghassemi@pmc.iaun.ac.ir

1 Department of Mechanical Engineering, Najafabad Branch, Islamic Azad University, Najafabad, Iran
${ }^{0} a_{i} \quad$ Base vector of convective coordinate system

${ }^{t} a_{i} \quad$ Base vector of convective coordinate system

${ }^{0}$ d Director vector

${ }^{t} \mathbf{d} \quad$ Director vector

$J=\frac{\mathrm{d}^{t} S}{\mathrm{~d}^{0} S} \quad$ Jacobean transformation

$F \quad$ Deformation gradient tensor

$w_{\text {ext }} \quad$ Virtual work of the external forces

$\bar{W} \quad$ Nondimensional deflection

$\overline{\sigma_{i}} \quad$ Nondimensional stress

$\phi \quad$ Skew angle

\section{Introduction}

Nonlinear deformation of plates and shells has been studied as an interesting and challenging subject in recent years. Skew plates are widely used in industrial modern structures such as decks of bridges, ship hulls, and buildings as reinforced slabs, stiffened, and decks (Shahidi et al. 2007). Deflection and stress distribution is one of the fundamental problems for designing of plates with large deformation. Von Karman is one of the most applicable nonlinear theories for large deformation of plates and shells which is 
suitable for moderately large deformation (Ugural 1981). This nonlinear theory does not have any mathematical complexities. Moreover, higher order shear deformation theory, considered Von Karman term, has been employed for large deformation of plates by many researchers. Derived equations have been solved by various numerical and analytical methods. Kumar et al. (2011) and Shi (2007) worked on bending analysis of plates using higher order shear deformation plate theory (HSDT) and nonlinear Von Karman theory analytically. Tahouneh and Naei (2016a, b) investigated the effect of bidirectional continuously graded nanocomposite materials on free vibration of thick shell panels rested on elastic foundations. They utilized 2-D differential quadrature method as an efficient numerical tool to discretize governing equations and implement boundary conditions. Their new results for natural frequencies of the shell included the effects of elastic coefficients of foundation, boundary conditions, and material and geometrical parameters. Moreover, the obtained results indicated that a graded nanocomposite volume fraction in two directions had a higher capability to reduce the natural frequency than the conventional 1-D functionally graded nanocomposite materials. They also analyzed free vibration and vibrational displacements of thick laminated curved panels with finite length resting on two-parameter elastic foundations based on the threedimensional elasticity theory. The effects of two-parameter elastic foundation modulus, and geometrical and material parameters together with the boundary conditions on the frequency parameters of the laminated functionally graded panels were investigated. They found that the outer functionally graded material layers have significant effect on the vibration behavior of cylindrical panels. Indeed, their study served as a benchmark for assessing the validity of numerical methods or two-dimensional theories used to analysis of laminated curved panels (Tahouneh and Naei 2016a, b). Tahouneh (2016) evaluated 3-D elasticity solution for free vibration analysis of continuously graded carbon nanotubereinforced rectangular plates resting on two-parameter elastic foundations. The volume fractions of oriented, straight single-walled carbon nanotubes (SWCNTs) were assumed to be graded in the thickness direction. Moreover, an equivalent continuum model based on the Eshelby-Mori-Tanaka approach was employed to estimate the effective constitutive law of the elastic isotropic medium with oriented, straight carbon nanotubes. A semi-analytical approach composed of differential quadrature method (DQM) and series solution was adopted to solve the equations of motion. Their novelty was to exploit Eshelby-Mori-Tanaka approach to reveal the impacts of the volume fractions of oriented CNTs, different CNTs distributions, various coefficients of foundation, and different combinations of free, simply supported, and clamped boundary conditions on the vibrational characteristics of CGCNTR rectangular plates. For numerical solution of this problem, Nguyen-Xuan et al. (2013), Shariyat (2010a) and Neff (2004) can be mentioned. Analytical solution of buckling and free vibration of plates using Von Karman theory studied by Fazzolari et al. (2013), Sahmani and Ansari (2013a) and Swaminathan and Naveenkumar (2014). Phung-Van et al. (2013), Sahmani and Ansari (2013b), Sahmani et al. (2014) and Shariyat (2010b) has used numerical method for solution of this problem. Amiri Rad and Panahandeh-Shahraki (2014), Duc and Cong (2013), Duc and Tung (2011), Lee and Kim (2014), Ovesy et al. (2012) and Yang et al. (2006) have applied HSDT and nonlinear Von Karman theory for post buckling analysis of plates. Cosserat brothers suggested Cosserat theory for the first time in 1909 (Cosserat and Cosserat 1909). Naghdi shell model according to Cosserat theory is another advanced theory for very large deformation of plates and shells (Simo 1993; Simo and Fox 1989; Simo et al. 1989, 1990a, b; Simo and Kennedy 1992). Some researchers have been solved large deflection of isotropic plates and shells with this theory by numerical methods (Ghassemi et al. 2010, 2011; Shahidi et al. 2007). Despite of accurate results, this theory has mathematical complexities in formulation and computation. For the first time, comparison of Cosserat and Von Karman theories has been studied in the present work. For this aim, large deflection and stress distribution of skew plates were obtained by two methods: first, HSDT by considering Von Karman nonlinearity and second, Cosserat theory. To consider the numerical solution, the whole domain method was employed. In the proposed method, the plate was considered as one element and the shape functions were developed up to very high order. The main purpose of this paper was to make a comparison between two theories from small to very large deflection of the skew plates under transverse loading. This comparison can give acceptable range of using Von Karman theory in large deflections. By determining this range, some large deflection analysis can be done using Von Karman as a simpler theory.

The outline of this paper is as follows. In "Kinematic equations of HSDT", the theory of higher order shear deformation plate by considering Von Karman nonlinearity is explained. The next section is about the kinematic relations of Cosserat theory followed by which numerical method is presented. The subsequent section gives the numerical results. Finally, conclusions are given.

\section{Kinematic equations of HSDT}

According to the HSDT, displacement field is considered as follows (Reddy 1984): 
$u_{1}\left(x_{1}, x_{2}, x_{3}\right)=u_{01}\left(x_{1}, x_{2}\right)+x_{3}\left[\Psi_{0 x_{1}}\left(x_{1}, x_{2}\right)-\frac{4}{3}\left(\frac{x_{3}}{h}\right)^{2}\left(\Psi_{0 x_{1}}\left(x_{1}, x_{2}\right)+\frac{\partial u_{03}}{\partial x_{1}}\right)\right]$
$u_{2}\left(x_{1}, x_{2}, x_{3}\right)=u_{02}\left(x_{1}, x_{2}\right)+x_{3}\left[\Psi_{0 x_{2}}\left(x_{1}, x_{2}\right)-\frac{4}{3}\left(\frac{x_{3}}{h}\right)^{2}\left(\Psi_{0 x_{2}}\left(x_{1}, x_{2}\right)+\frac{\partial u_{03}}{\partial x_{2}}\right)\right]$
$u_{3}\left(x_{1}, x_{2}, x_{3}\right)=u_{03}\left(x_{1}, x_{2}\right)$,

where $h$ is the thickness of the plate, and $u_{01}$ and $u_{02}$ are midplane displacements in $x_{1}$ and $x_{2}$ directions, respectively.

$u_{03}$ is displacement of plate in $x_{3}$ direction. $\Psi_{0 x_{1}}$ and $\Psi_{0 x_{2}}$ are the midplane rotation of the normal around the $x_{1}$ and $x_{2}$ axes. Figure 1 shows the geometry of applied rectangular plate.

where

$k_{1}=k_{1}^{0}+x_{3}^{2} k_{1}^{2}$

$k_{2}=k_{2}^{0}+x_{3}^{2} k_{2}^{2}$

$k_{6}=k_{6}^{0}+x_{3}^{2} k_{6}^{2}$.

Strain field can be achieved by displacement in Eq. (2). It is notable that Von Karman theory is added due to large deformation:

$\varepsilon_{1}=\varepsilon_{1}^{0}+x_{3}\left(\kappa_{1}^{0}+x_{3}^{2} \kappa_{1}^{2}\right), \quad \varepsilon_{2}=\varepsilon_{2}^{0}+x_{3}\left(\kappa_{2}^{0}+x_{3}^{2} \kappa_{2}^{2}\right), \quad \varepsilon_{3}=0$

$\varepsilon_{4}=\varepsilon_{4}^{0}+x_{3}^{2} \kappa_{4}^{2}, \quad \varepsilon_{5}=\varepsilon_{5}^{0}+x_{3}^{2} \kappa_{5}^{2}, \quad \varepsilon_{6}=\varepsilon_{6}^{0}+x_{3}\left(\kappa_{6}^{0}+x_{3}^{2} \kappa_{6}^{2}\right)$,

The stress resultants and couple stresses are given by Eq. (7):

$\left(N_{i}, M_{i}, P_{i}\right)=\int_{-h / 2}^{+h / 2} \sigma_{i}\left(1, x_{3}, x_{3}^{3}\right) \mathrm{d} x_{3} \quad i=1,2,6$

where

$\varepsilon_{1}^{0}=\frac{\partial u_{01}}{\partial x_{1}}+\frac{1}{2}\left(\frac{\partial u_{03}}{\partial x_{1}}\right)^{2}, \quad \varepsilon_{2}^{0}=\frac{\partial u_{02}}{\partial x_{2}}+\frac{1}{2}\left(\frac{\partial u_{03}}{\partial x_{2}}\right)^{2}, \quad \varepsilon_{4}^{0}=\Psi_{0 x_{2}}+\frac{\partial u_{03}}{\partial x_{2}}$

$\varepsilon_{5}^{0}=\Psi_{0 x_{1}}+\frac{\partial u_{03}}{\partial x_{1}}, \quad \varepsilon_{6}^{0}=\frac{\partial u_{01}}{\partial x_{2}}+\frac{\partial u_{02}}{\partial x_{1}}+\frac{\partial u_{03}}{\partial x_{1}} \frac{\partial u_{03}}{\partial x_{2}}$.

Midplane curvatures in Eq. (2) are as follows:

$\kappa_{1}^{0}=\frac{\Psi_{0 x_{1}}}{\partial x_{1}}, \quad \kappa_{2}^{0}=\frac{\Psi_{0 x_{2}}}{\partial x_{2}}, \quad \kappa_{6}^{0}=\frac{\Psi_{0 x_{1}}}{\partial x_{2}}+\frac{\Psi_{0 x_{2}}}{\partial x_{1}}$

$\kappa_{1}^{2}=-\frac{4}{3 h^{2}}\left(\frac{\Psi_{0 x_{1}}}{\partial x_{1}}+\frac{\partial^{2} u_{03}}{\partial x_{1}^{2}}\right), \quad \kappa_{2}^{2}=-\frac{4}{3 h^{2}}\left(\frac{\Psi_{0 x_{2}}}{\partial x_{2}}+\frac{\partial^{2} u_{03}}{\partial x_{2}^{2}}\right)$

$\kappa_{4}^{2}=-\frac{4}{h^{2}}\left(\Psi_{0 x_{2}}+\frac{\partial u_{03}}{\partial x_{2}}\right), \quad \kappa_{5}^{2}=-\frac{4}{h^{2}}\left(\Psi_{0 x_{1}}+\frac{\partial u_{03}}{\partial x_{1}}\right)$

$\kappa_{6}^{2}=-\frac{4}{3 h^{2}}\left(\frac{\Psi_{0 x_{1}}}{\partial x_{2}}+\frac{\Psi_{0 x_{2}}}{\partial x_{1}}+2 \frac{\partial^{2} u_{03}}{\partial x_{1} \partial x_{2}}\right)$.

For an elastic isotropic material, constitutive equations are as follows:

$$
\begin{aligned}
& \sigma_{1}=\frac{E}{1-v^{2}}\left(\varepsilon_{1}^{0}+v \varepsilon_{2}^{0}+x_{3}\left[k_{1}+v k_{2}\right]\right) \\
& \sigma_{2}=\frac{E}{1-v^{2}}\left(\varepsilon_{2}^{0}+v \varepsilon_{1}^{0}+x_{3}\left[k_{2}+v k_{1}\right]\right) \\
& \sigma_{4}=\frac{E}{1-v^{2}}\left(\frac{1-v}{2}\right)\left(\varepsilon_{4}^{0}+x_{3}^{2} k_{4}^{2}\right) \\
& \sigma_{5}=\frac{E}{1-v^{2}}\left(\frac{1-v}{2}\right)\left(\varepsilon_{5}^{0}+x_{3}^{2} k_{5}^{2}\right) \\
& \sigma_{6}=\frac{E}{1-v^{2}}\left(\frac{1-v}{2}\right)\left(\varepsilon_{6}^{0}+x_{3} k_{6}\right),
\end{aligned}
$$$$
\left(Q_{2}, R_{2}\right)=\int_{-h / 2}^{+h / 2} \sigma_{4}\left(1, x_{3}^{2}\right) \mathrm{d} x_{3}, \quad\left(Q_{1}, R_{1}\right)=\int_{-h / 2}^{+h / 2} \sigma_{5}\left(1, x_{3}^{2}\right) \mathrm{d} x_{3},
$$

where $N_{i}$ and $Q_{i}$ are the membrane and transverse shear forces and $M_{i}$ is the bending moment per unit length. $P_{i}$ and $R_{i}$ are the higher order bending moment and shear force, respectively.

Therefore, the constitutive relations of the plate can be rewritten by substituting Eq. (5) into Eq. (7) as follows:

$\left[\begin{array}{l}N \\ M \\ P\end{array}\right]=\left[\begin{array}{lll}A & B & E \\ B & D & F \\ E & F & H\end{array}\right]\left[\begin{array}{l}\varepsilon^{0} \\ \kappa^{0} \\ \kappa^{2}\end{array}\right]$

$\left[\begin{array}{l}\bar{Q} \\ \bar{R}\end{array}\right]=\left[\begin{array}{ll}A & D \\ D & F\end{array}\right]\left[\begin{array}{l}\varepsilon^{0} \\ \kappa^{2}\end{array}\right]$

where the matrix coefficients are as follows: 


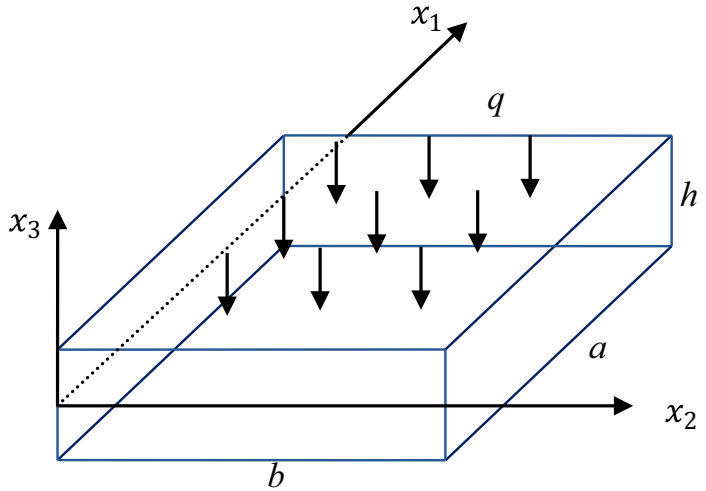

Fig. 1 Geometry of rectangular plate subject to uniform load
For numerical solution of Eq. (10), let displacement vector as follows:

$\mathbf{U}=\left[\begin{array}{l}u_{1} \\ u_{2} \\ u_{3} \\ \psi_{x_{1}} \\ \psi_{x_{2}}\end{array}\right]$.

This displacement vector can be discretized as follows:

$\mathbf{U}=\mathbf{S} \widehat{\mathbf{x}}$,

where $\mathbf{S}$ is the shape function matrix and $\widehat{\mathbf{x}}$ is the general coordinate system. To determine parameter $\hat{\mathbf{x}}$, the whole domain method was employed which is explained in "Numerical method".

$\left(A_{i j}, B_{i j}, D_{i j}, E_{i j}, F_{i j}, H_{i j}\right)=\int_{-h / 2}^{+h / 2}\left(Q_{i j}\right)\left(1, x_{3}, x_{3}^{2}, x_{3}^{3}, x_{3}^{4}, x_{3}^{6}\right) \mathrm{d} x_{3} \quad i, j=1,2,6$

$\left(A_{i j}, D_{i j}, F_{i j}\right)=\int_{-h / 2}^{+h / 2}\left(Q_{i j}\right)\left(1, x_{3}^{2}, x_{3}^{4}\right) \mathrm{d} x_{3} \quad i, j=4,5$.

The principle of virtual work is as follows:

$\delta U+\delta V=0$,

where $\delta U$ is the virtual strain energy and this term can be written as follows:

$\delta U=\iiint_{-\frac{h}{2}}^{+\frac{h}{2}}\left(\sigma_{i} \delta \varepsilon_{i}\right) \mathrm{d} x_{1} \mathrm{~d} x_{2} \mathrm{~d} x_{3} \quad i=1,2,4,5,6$.

$\delta V$ is the virtual work done by external forces and moments.

According to Eq. (7), virtual strain energy can be rewritten as follows:

\section{Kinematic relations of Cosserat theory}

Figure 2 shows an element of a plate before and after deformation. Superscripts " 0 " and " $t$ " are implied as the status of "before deformation" and "after deformation" respectively. ${ }^{0} a_{i}$ and ${ }^{t} a_{i}$ are the base vectors of convective coordinate system. In addition, in this figure, ${ }^{0} \mathbf{d}$ and ${ }^{t} \mathbf{d}$ are the director vectors. These vectors are considered normal to the midplane status.

The base vectors in Current configuration can be written as follows (Simo et al. 1989):

${ }^{t} \mathbf{a}_{\alpha}={ }^{t} \mathbf{X}_{, \alpha},{ }^{t} \mathbf{a}_{3}={ }^{t} \mathbf{a}^{3}=\frac{{ }^{t} \mathbf{a}_{1} \times{ }^{t} \mathbf{a}_{2}}{\left\|{ }^{t} \mathbf{a}_{1} \times{ }^{t} \mathbf{a}_{2}\right\|}$.

Using Eq. (15), the first and second fundamental tensors of the surface can be written as follows:

$\delta U=\iint\left(N_{i} \delta \varepsilon_{i}^{0}+M_{i} \delta \kappa_{i}^{0}+P_{i} \delta \kappa_{i}^{2}+Q_{1} \delta \varepsilon_{5}^{0}+Q_{2} \delta \varepsilon_{4}^{0}+R_{1} \delta \kappa_{5}^{2}+R_{2} \delta \kappa_{4}^{2}\right) \mathrm{d} x_{1} \mathrm{~d} x_{2} \quad i=1,2,6$.

Fig. 2 An element of plate before and after deformation. a Reference configuration. b Current configuration

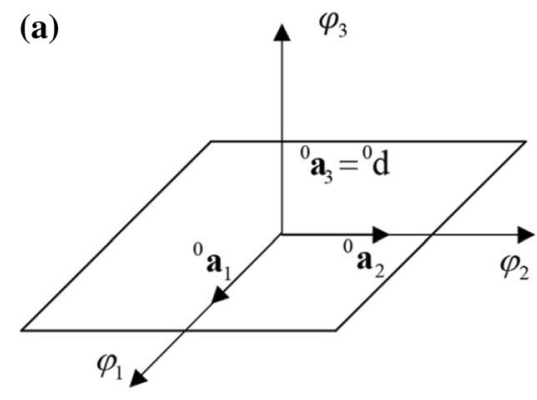

Reference configuration

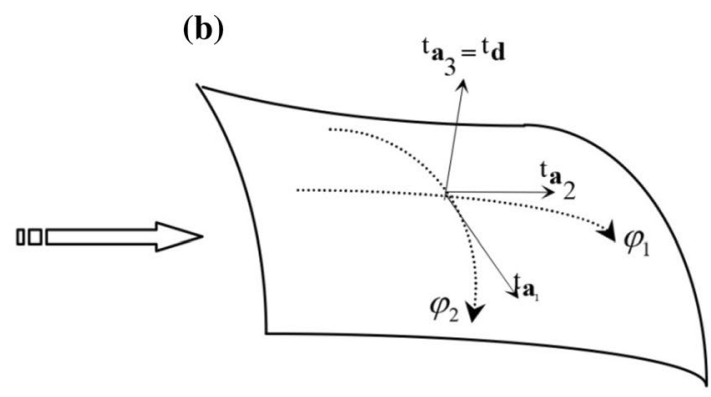

Current configuration 
${ }^{t} a_{\alpha \beta}={ }^{t} \mathbf{x}_{, \alpha} \cdot{ }^{t} \mathbf{x}_{, \beta} \quad{ }^{t} b_{\alpha \beta}=-{ }^{t} \mathbf{a}_{3, \alpha} \cdot{ }^{t} \mathbf{a}_{\beta}={ }^{t} \mathbf{a}_{3} \cdot{ }^{t} \mathbf{a}_{\alpha, \beta}$

By this definition membrane and bending strains are expressed as follows:

${ }_{0}^{t} \varepsilon_{\alpha \beta}=\frac{1}{2}\left({ }^{t} \mathbf{x}_{, \alpha} \cdot{ }^{t} \mathbf{x}_{, \beta}-{ }^{0} \mathbf{x}_{, \alpha} \cdot{ }^{0} \mathbf{x}_{, \beta}\right)$

${ }^{t} \kappa_{\alpha \beta}={ }^{t} b_{\alpha \beta}={ }^{t} \mathbf{a}_{3} \cdot{ }^{t} \mathbf{x}_{, \alpha \beta}=\frac{1}{\sqrt{{ }^{t} a}}\left[{ }^{t} \mathbf{x}_{, \alpha \beta} \cdot{ }^{t} \mathbf{x}_{, 1} \times{ }^{t} \mathbf{x}_{2,}\right]$

${ }_{0}^{t} \kappa_{\alpha \beta}={ }^{t} \kappa_{\alpha \beta}-{ }^{0} \kappa_{\alpha \beta}$.

In Eq. (17), subscript "0" denotes reference configuration.

According to the above equation, consider strain vector, ${ }_{0}^{t} \boldsymbol{\varepsilon}$, as:

${ }_{0}^{t} \varepsilon=\left[{ }_{0}^{t} \varepsilon_{11},{ }_{0}^{t} \varepsilon_{22}, 2{ }_{0}^{t} \varepsilon_{12},{ }_{0}^{t} \kappa_{11},{ }_{0}^{t} \kappa_{22}, 2{ }_{0}^{t} \kappa_{12}\right]$.

Considering Naghdi shell model, invariant form of Cauchy stress resultants is obtained as follows:
In Eq. (21), $J=\frac{\mathrm{d}^{\prime} S}{\mathrm{~d}^{0} S}$ is the Jacobean transformation. This term is the ratio between the element surface after and before deformation:

$J=\operatorname{det}(\mathbf{F}) \quad$ where $\mathrm{F}_{i}^{j}={ }^{t} \mathbf{a}_{i} \otimes{ }^{0} \mathbf{a}^{j}={ }^{t} \mathbf{x}_{, i} \otimes{ }^{0} \mathbf{x}_{, j}$.

In Eq. (22), " $F$ " is the deformation gradient tensor.

According to Eq. (20), the resultant stress vector can be defined as follows:

${ }_{0}^{t} \boldsymbol{\sigma}=\left[{ }_{0}^{t} N^{11},{ }_{0}^{t} N^{22},{ }_{0}^{t} N^{12},{ }_{0}^{t} M^{11},{ }_{0}^{t} M^{22},{ }_{0}^{t} M^{12}\right]$.

Based on the Hooke's law for an isotropic linear elastic material, resultant stress and strain vectors are related as follows (Simo and Kennedy 1992):

${ }_{0}^{t} \boldsymbol{\sigma}=\mathbf{D}_{0}^{t} \boldsymbol{\varepsilon}$,

where:

$\mathbf{D}=\left[\begin{array}{cc}\mathbf{D}_{\mathbf{m}} & \mathbf{0} \\ \mathbf{0} & \mathbf{D}_{\mathbf{b}}\end{array}\right]$

$$
\begin{aligned}
& { }_{t}^{t} N={ }_{t}^{t} N^{\alpha \beta t} \mathbf{a}_{\alpha} \otimes{ }^{t} \mathbf{a}_{\beta} \\
& { }_{t}^{t} Q={ }_{t}^{t} Q^{\alpha t} \mathbf{a}_{\alpha} \\
& { }_{t}^{t} M={ }_{t}^{t} M^{\alpha \beta t} \mathbf{a}_{\alpha} \otimes{ }^{t} \mathbf{a}_{\beta} .
\end{aligned}
$$

In Eq. (19), subscript " $t$ " denotes current configuration. In the initial configuration, second Piola stress resultants can be written as follows:

$$
\begin{aligned}
& { }_{0}^{t} N={ }_{0}^{t} N^{\alpha \beta 0} \mathbf{a}_{\alpha} \otimes{ }^{0} \mathbf{a}_{\beta} \\
& { }_{0}^{t} Q={ }_{0}^{t} Q^{\alpha \beta 0} \mathbf{a}_{\alpha} \otimes{ }^{0} \mathbf{a}_{\beta} \\
& { }_{0}^{t} M={ }_{0}^{t} M^{\alpha \beta 0} \mathbf{a}_{\alpha} \otimes{ }^{0} \mathbf{a}_{\beta} .
\end{aligned}
$$

In Eq. (20), subscript "0" denotes reference configuration. The Cauchy and Piola stresses are related as follows:

${ }_{0}^{t} N^{\alpha \beta}=J_{t}^{t} N^{\alpha \beta}, \quad{ }_{0}^{t} M^{\alpha \beta}=J_{t}^{t} M^{\alpha \beta}$.
Principle of virtual work can be used for equilibrium condition in current and reference configuration. These equations are written as follows:

Current configuration : $\int_{{ }_{t} S}\left({ }_{t}^{t} N^{\alpha \beta} \delta_{0}^{t} \varepsilon_{\alpha \beta}+{ }_{t}^{t} M^{\alpha \beta} \delta_{0}^{t} \kappa_{\alpha \beta}\right) \mathrm{d}{ }^{t} S={ }^{t} W_{\mathrm{ext}}$

Reference configuration : $\int_{{ }_{0 S}}\left({ }_{0}^{t} N^{\alpha \beta} \delta_{0}^{t} \varepsilon_{\alpha \beta}+{ }_{0}^{t} M^{\alpha \beta} \delta_{0}^{t} \kappa_{\alpha \beta}\right) \mathrm{d}^{0} S={ }^{t} W_{\text {ext }}$.

$W_{\text {ext }}$ is virtual work of the external forces. It can be written as follows:

$\int_{{ }^{i} S}\left(\mathbf{F}_{\text {ext }} \cdot \delta^{t} \mathbf{U}+\mathbf{M}_{\text {ext }} \cdot \delta^{t} \mathbf{d}\right) \mathrm{d}^{t} S={ }^{t} W_{\text {ext }}$.

For numerical solution of Eq. (27) or (28), displacement vector should be discretized by considering

${ }^{t} \mathbf{X}={ }^{\mathbf{0}} \mathbf{X}+\mathbf{U}$. 
Displacement vector can be discretized as follows:

$\mathbf{U}=\widehat{\mathbf{S}}$,

where $\mathbf{S}$ is the shape function matrix and $\widehat{\mathbf{X}}$ is the general coordinate system.

So:

$\delta^{t} e_{\alpha \beta}=\left(\mathbf{x}_{, \alpha}^{\mathrm{T}} \mathbf{s}_{, \beta}+\mathbf{x}_{, \beta}^{\mathrm{T}} \mathbf{s}_{, \alpha}\right) \delta \hat{\mathbf{X}}=\mathbf{E}_{\alpha \beta} \delta \hat{\mathbf{X}}$

$\delta^{t} \kappa_{\alpha \beta}=\left(\frac{1}{\sqrt{{ }^{t} a}} \Gamma_{\boldsymbol{\alpha} \boldsymbol{\beta}}-\frac{\gamma_{\alpha \beta}}{2 \sqrt{{ }^{t} a^{3}}} \mathbf{A}\right) \delta \hat{\mathbf{X}}=\mathbf{K}_{\alpha \beta} \delta \hat{\mathbf{X}}$

$\delta^{t} \mathbf{d}=\delta^{t} \mathbf{a}_{3}=\left(\frac{1}{\sqrt{{ }^{t} a}} \boldsymbol{\beta}_{12}^{\mathrm{T}}-\frac{1}{2 \sqrt{{ }^{t} a^{3}}} \mathbf{A}^{\mathrm{T}}\left(x_{, 1} \times x_{, 2}\right)^{\mathrm{T}}\right) \delta \hat{\mathbf{X}}=\mathbf{Y} \delta \hat{\mathbf{X}}$,

where

$\boldsymbol{\Gamma}_{\alpha \beta}=\left({ }^{t} \boldsymbol{X}_{, 1} \times{ }^{t} \boldsymbol{X}_{, 2}\right){ }^{\mathrm{T}} \mathbf{S}_{, \alpha \beta}+\left({ }^{t} \boldsymbol{X}_{, 2} \times{ }^{t} \boldsymbol{X}_{, \alpha \beta}\right)^{\mathrm{T}} \mathbf{S}_{, 1}+\left({ }^{t} \boldsymbol{X}_{, \alpha \beta} \times{ }^{t} \boldsymbol{X}_{, 1}\right)^{\mathrm{T}} \mathbf{S}_{, 2}$

$\boldsymbol{\gamma}_{\alpha \beta}={ }^{t} \boldsymbol{X}_{, \alpha \beta} \cdot{ }^{t} \boldsymbol{X}_{, 1} \times{ }^{t} \boldsymbol{X}_{, 2}$.

$\mathbf{A}=2^{t} a_{11}{ }^{t} X_{, 2}^{\mathrm{T}} \cdot S_{, 2}+2^{t} a_{22}{ }^{t} X_{, 1}^{\mathrm{T}} \cdot S_{, 1}-2^{t} a_{12}{ }^{t} X_{, 2}^{\mathrm{T}} \cdot S_{, 1}-2^{t} a_{12}{ }^{t} X_{, 1}^{\mathrm{T}} \cdot S_{, 2}$

$\boldsymbol{\beta}_{12}=\left[\begin{array}{ccc}\mathbf{0} & { }^{t} x_{3,2} S_{2,1}-{ }^{t} x_{3,1} S_{2,2}{ }^{t} x_{2,1} S_{3,2}-{ }^{t} x_{2,2} S_{3,1} \\ { }^{t} x_{3,1} S_{1,2}-{ }^{t} x_{3,2} S_{1,1} & \mathbf{0} & { }^{t} x_{1,2} S_{3,1}-{ }^{t} x_{1,1} S_{3,2} \\ { }^{t} x_{2,2} S_{1,1}-{ }^{t} x_{2,1} S_{1,2} & { }^{t} x_{1,1} S_{2,2}-{ }^{t} x_{1,2} S_{2,1} & \mathbf{0}\end{array}\right]$.

Fig. 3 Mapping an arbitrary skew plate into the standard

square computational domain
By substitution of Eqs. (32)-(36) in Eq. (28), the following relation can be achieved:

$\int_{{ }^{0} S}{ }_{0}^{t} N^{\alpha \beta} \mathbf{E}_{\alpha \beta}+{ }_{0}^{t} M^{\alpha \beta} \mathbf{K}_{\alpha \beta} \mathrm{d}{ }^{0} S=\int_{{ }^{t} S}{ }_{t}^{t} \mathbf{F}_{\mathrm{ext}}^{\mathrm{T}} \mathbf{S}+{ }_{t}^{t} \mathbf{M}_{\mathrm{ext}}^{\mathrm{T}} \mathbf{Y} \mathrm{d}{ }^{t} S$,

where $\mathbf{E}_{\alpha \beta}, \mathbf{K}_{\alpha \beta}$, and $\mathbf{Y}$ would be determined through the Eqs. (32)-(34), respectively.

\section{Numerical method}

Whole domain method was applied for numerical solution of virtual work equations for both theories. In this method, whole domain of the plate will be considered as one element. First, the domain of the plate is mapped to a standard square (Fig. 3).

Hierarchical finite-element shape function was employed for interpolation of displacement field. Equations (40)-(42) show shape functions of free, simply supported, and clamped boundary conditions in one direction:

1. Free

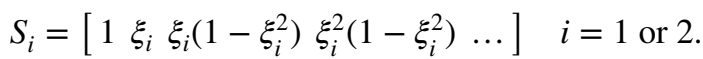

2. Simply support

$$
S_{i}=\left[1-\xi_{i}^{2} \xi_{i}\left(1-\xi_{i}^{2}\right) \xi_{i}^{2}\left(1-\xi_{i}^{2}\right) \xi_{i}^{3}\left(1-\xi_{i}^{2}\right) \ldots\right] \quad i=1 \text { or } 2 .
$$

$x_{2}$

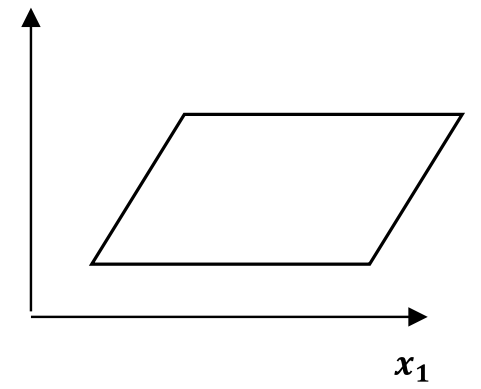

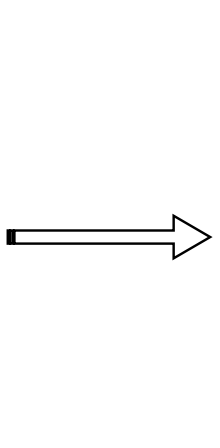

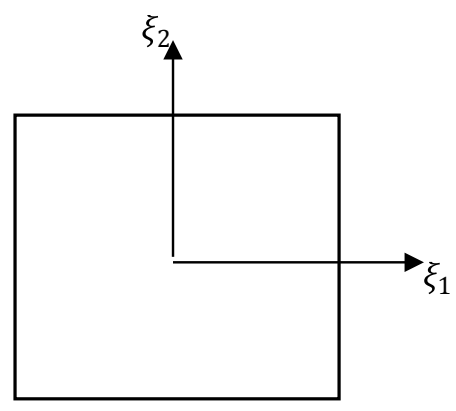

Fig. 4 Roller boundary condition of rectangular plate under Sinusoidal load
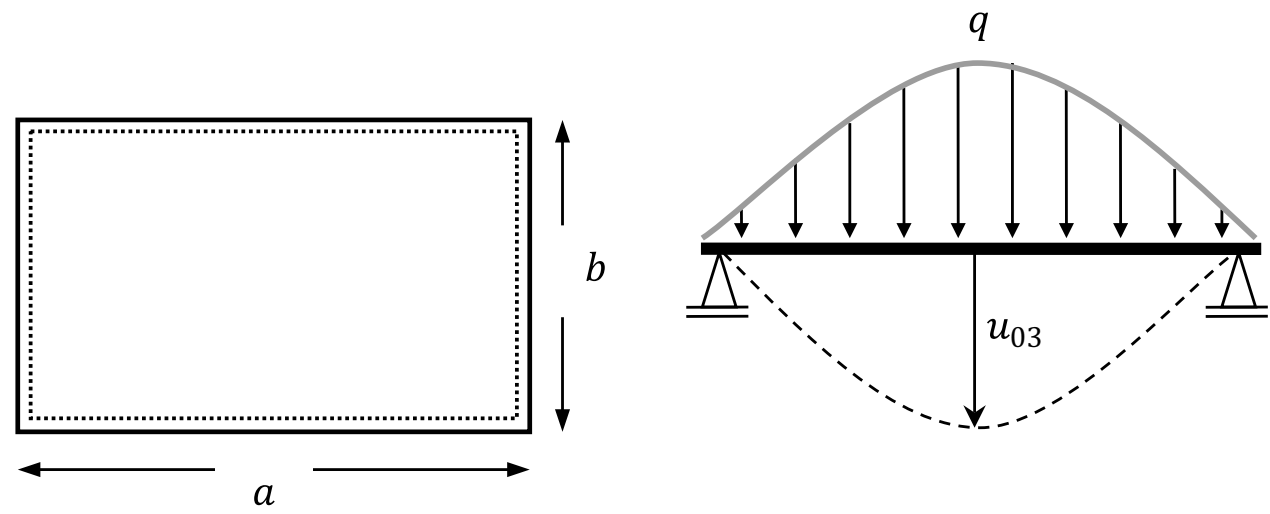
3. Clamped

$S_{i}=\left[\left(1-\xi_{i}^{2}\right)^{2} \xi_{i}\left(1-\xi_{i}^{2}\right)^{2} \xi_{i}^{2}\left(1-\xi_{i}^{2}\right)^{2} \xi_{i}^{3}\left(1-\xi_{i}^{2}\right)^{2} \ldots\right] \quad i=1$ or 2.

For two-dimensional cases, shape functions matrix is as follows:

$S=S_{1} S_{2}^{\mathrm{T}}$.

Therefore, displacement in each direction can be discretized as follows:

$U_{i}=S_{i} \hat{X}$

\section{Numerical results}

To validate the present numerical method, the results of the two theories are compared separately with the literatures. Then, the difference between two theories is compared to rectangular and skew plates from small to very large deformation.

\section{Validation the numerical result of HSDT by consideration Von Karman nonlinearity}

In this section, HSDT which is solved by whole domain method is compared with exact solution presented by

Table 1 Comparison of the nondimensional deflection at the center of a rectangular plate under distributed load

\begin{tabular}{|c|c|c|c|c|c|c|c|c|c|c|c|}
\hline \multirow[t]{2}{*}{$\frac{a}{h}$} & \multicolumn{3}{|l|}{$\bar{W}$} & \multirow{2}{*}{$\frac{a}{h}$} & \multicolumn{3}{|l|}{$\bar{W}$} & \multirow{2}{*}{$\frac{a}{h}$} & \multicolumn{3}{|l|}{$\bar{W}$} \\
\hline & $\begin{array}{l}\text { Mechab } \\
\text { et al. } \\
(2010)\end{array}$ & Present model & Error (\%) & & $\begin{array}{l}\text { Mechab } \\
\text { et al. } \\
(2010)\end{array}$ & Present model & Error (\%) & & $\begin{array}{l}\text { Mechab } \\
\text { et al. } \\
(2010)\end{array}$ & Present model & Error $(\%)$ \\
\hline 2 & 0.6993 & 0.7371 & 5.4078 & 8 & 0.3047 & 0.3256 & 6.8494 & 14.5 & 0.2890 & 0.2954 & 2.2366 \\
\hline 2.5 & 0.5733 & 0.5943 & 3.6577 & 8.5 & 0.3084 & 0.3205 & 3.9481 & 15 & 0.2890 & 0.2944 & 1.8742 \\
\hline 3 & 0.5213 & 0.5073 & 2.6791 & 9 & 0.2894 & 0.3155 & 9.0158 & 15.5 & 0.2890 & 0.2935 & 1.5713 \\
\hline 3.5 & 0.4588 & 0.4623 & 0.7596 & 9.5 & 0.2878 & 0.3122 & 8.4606 & 16 & 0.2852 & 0.2927 & 2.6336 \\
\hline 4 & 0.4068 & 0.4243 & 4.2961 & 10 & 0.2863 & 0.3089 & 7.8965 & 16.5 & 0.2702 & 0.2920 & 8.0825 \\
\hline 4.5 & 0.3792 & 0.3967 & 4.6061 & 10.5 & 0.2847 & 0.3073 & 7.9414 & 17 & 0.2710 & 0.2911 & 7.4115 \\
\hline 5 & 0.3552 & 0.3831 & 7.8684 & 11 & 0.2849 & 0.3075 & 7.9365 & 17.5 & 0.2711 & 0.2904 & 7.1533 \\
\hline 5.5 & 0.3416 & 0.3660 & 7.1588 & 11.5 & 0.2794 & 0.3042 & 8.8869 & 18 & 0.2711 & 0.2898 & 6.8954 \\
\hline 6 & 0.3385 & 0.3525 & 4.1308 & 12 & 0.2904 & 0.3026 & 4.1916 & 18.5 & 0.2711 & 0.2892 & 6.7014 \\
\hline 6.5 & 0.3249 & 0.3494 & 7.5294 & 13 & 0.2889 & 0.2994 & 3.6275 & 19 & 0.2711 & 0.2887 & 6.5078 \\
\hline 7 & 0.3161 & 0.3374 & 6.7402 & 13.5 & 0.2889 & 0.2977 & 3.0224 & 19.5 & 0.2711 & 0.2866 & 5.7344 \\
\hline 7.5 & 0.3063 & 0.3306 & 7.9501 & 14 & 0.2890 & 0.2965 & 2.5994 & 20 & 0.2713 & 0.2795 & 3.0263 \\
\hline
\end{tabular}

Table 2 Comparison of the nondimensional stress at the center of a rectangular plate for two aspect ratios

\begin{tabular}{|c|c|c|c|c|c|c|c|c|c|}
\hline \multirow[t]{2}{*}{$a / b$} & \multirow[t]{2}{*}{$\overline{x_{3}}=\frac{x_{3}}{h}$} & \multicolumn{3}{|l|}{$\bar{\sigma}_{y}$} & \multirow[t]{2}{*}{$a / b$} & \multirow[t]{2}{*}{$\overline{x_{3}}=\frac{x_{3}}{h}$} & \multicolumn{3}{|l|}{$\bar{\sigma}_{y}$} \\
\hline & & Mechab et al. (2010) & Present model & Error $(\%)$ & & & Mechab et al. (2010) & Present model & Error $(\%)$ \\
\hline \multirow[t]{13}{*}{2} & -0.50 & -0.6522 & -0.6430 & 1.4061 & 3 & -0.50 & -0.3478 & -0.3390 & 2.5386 \\
\hline & -0.40 & -0.5870 & -0.5790 & 1.3561 & & -0.40 & -0.3261 & -0.3180 & 2.4809 \\
\hline & -0.35 & -0.5435 & -0.5400 & 0.6403 & & -0.35 & -0.2826 & -0.2740 & 3.0466 \\
\hline & -0.25 & -0.4783 & -0.4690 & 1.9362 & & -0.25 & -0.2609 & -0.2520 & 3.4002 \\
\hline & -0.15 & -0.4130 & -0.4080 & 1.2202 & & -0.15 & -0.2391 & -0.2260 & 5.4907 \\
\hline & 0.00 & -0.2609 & -0.2570 & 1.4835 & & 0.00 & -0.1304 & -0.1280 & 1.8706 \\
\hline & 0.10 & -0.1739 & -0.1690 & 2.8233 & & 0.10 & -0.0435 & -0.0410 & 5.7038 \\
\hline & 0.25 & 0.2391 & 0.2270 & 5.0726 & & 0.25 & 0.1739 & 0.1660 & 4.5483 \\
\hline & 0.30 & 0.4348 & 0.4290 & 1.3294 & & 0.30 & 0.3043 & 0.2920 & 4.0547 \\
\hline & 0.35 & 0.6957 & 0.6880 & 1.0997 & & 0.35 & 0.4783 & 0.4660 & 2.5635 \\
\hline & 0.40 & 1.0000 & 1.0800 & 8.0000 & & 0.40 & 0.6087 & 0.5940 & 2.4134 \\
\hline & 0.45 & 1.5870 & 1.5740 & 0.8167 & & 0.45 & 0.7826 & 0.7760 & 0.8433 \\
\hline & 0.50 & 1.9565 & 1.9490 & 0.3844 & & 0.50 & 1.0870 & 1.0710 & 1.4683 \\
\hline
\end{tabular}


Mechab et al. (2010). To this aim, consider a rectangular plate with roller boundary conditions as follows (Fig. 4):

$u_{3}(x, b)=u_{3}(x, 0)=u_{3}(a, y)=u_{3}(0, y)$

$u_{2}(0, y)=u_{2}(a, y)=0$

$u_{1}(x, b)=u_{1}(x, 0)=0$.

The Young's modulus of the plate is $E=2 \times 10^{11}(\mathrm{~Pa})$ and the poisson ratio $v=0.3$. Transverse loading $q$ is considered as follows: $q=q_{0} \sin \frac{\pi x}{a} \sin \frac{\pi y}{b}$

The dimensionless formulation of deflection and stresses are as follows:

$\bar{W}=\frac{10 h^{3} E}{a^{4} q_{0}} u_{3}\left(\frac{a}{2}, \frac{b}{2}\right), \quad \bar{\sigma}=\frac{h}{a q_{0}} \sigma_{i}\left(\frac{a}{2}, \frac{b}{2}, \frac{h}{3}\right)$.

Table 1 reveals the nondimensional deflection at the center of a rectangular plate for various thicknesses. As
Fig. 5 Simply supported boundary condition of rectangular plate under uniform load
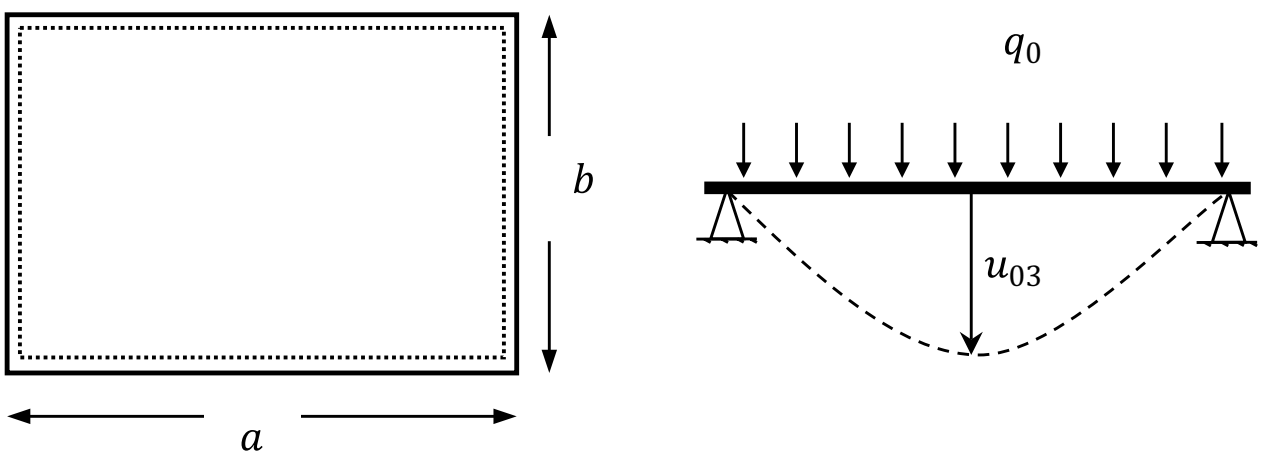

Table 3 Comparison of the nondimensional deflection at the center of a rectangular plate under distributed load

\begin{tabular}{|c|c|c|c|c|c|c|c|}
\hline \multirow{2}{*}{$\frac{q_{0} a^{4}}{D h} \times 10^{-6}$} & \multicolumn{3}{|l|}{$\bar{W}$} & \multirow{2}{*}{$\frac{q_{0} a^{2}}{D h} \times 10^{-6}$} & \multicolumn{3}{|l|}{$\overline{\bar{W}}$} \\
\hline & Shahidi et al. (2007) & Present model & Error $(\%)$ & & Shahidi et al. (2007) & Present model & Error $(\%)$ \\
\hline 0 & 3.7975 & 3.7973 & 0.0045 & 10 & 28.0576 & 28.4550 & 1.4164 \\
\hline 0.5 & 11.6429 & 11.8497 & 1.7762 & 12 & 28.8104 & 29.8691 & 3.6747 \\
\hline 1 & 13.6655 & 14.0583 & 2.8744 & 14 & 29.8150 & 31.4102 & 5.3503 \\
\hline 2 & 15.6881 & 16.1039 & 2.6504 & 16 & 30.8189 & 31.9304 & 3.6066 \\
\hline 3 & 17.9613 & 18.1174 & 0.8691 & 20 & 32.3225 & 33.3278 & 3.1102 \\
\hline 4 & 19.9832 & 20.6076 & 3.1246 & 25 & 35.3376 & 36.3831 & 2.9586 \\
\hline 5 & 21.7513 & 22.1562 & 1.8615 & 30 & 37.3441 & 38.5839 & 3.3199 \\
\hline 6 & 23.7720 & 24.2320 & 1.9350 & 35 & 39.8550 & 41.7791 & 4.8278 \\
\hline 7 & 25.2862 & 25.5262 & 0.9491 & 40 & 42.1126 & 43.7732 & 3.9432 \\
\hline 8 & 26.7999 & 26.8560 & 0.2093 & 50 & 47.3847 & 48.5269 & 2.4105 \\
\hline
\end{tabular}

Fig. 6 Simply supported boundary condition of rectangular plate under concentrated load

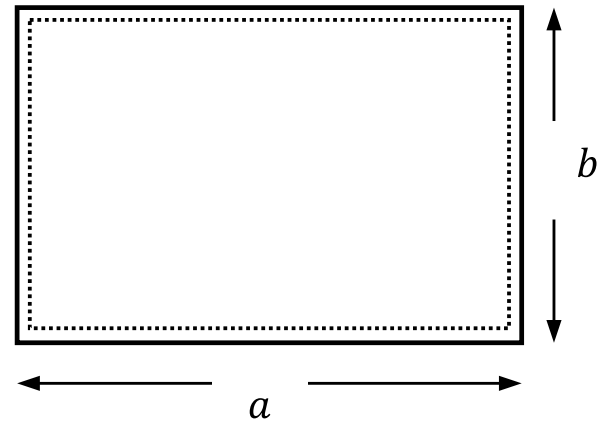


Table 4 Comparison of the center deflection for a rectangular plate under concentrated load

\begin{tabular}{|c|c|c|c|c|c|c|c|}
\hline \multirow{2}{*}{$\frac{q_{0} a^{4}}{D h} \times 10^{-6}$} & \multicolumn{3}{|l|}{$\bar{W}$} & \multirow{2}{*}{$\frac{q_{0} a^{2}}{D h} \times 10^{-6}$} & \multicolumn{3}{|l|}{$\overline{\bar{W}}$} \\
\hline & Shahidi et al. (2007) & Present model & Error $(\%)$ & & Shahidi et al. (2007) & Present model & Error $(\%)$ \\
\hline 1 & 17.0950 & 18.1126 & 5.9526 & 24 & 62.6816 & 63.5268 & 1.3484 \\
\hline 1.5 & 22.7933 & 23.8332 & 4.5623 & 29 & 67.3743 & 67.607 & 0.3454 \\
\hline 3.5 & 28.4916 & 29.3005 & 2.8391 & 35 & 71.3966 & 72.0926 & 0.9748 \\
\hline 6 & 34.1899 & 35.6996 & 4.4156 & 43 & 76.4246 & 77.0712 & 0.8461 \\
\hline 7.5 & 40.2235 & 41.1421 & 2.2837 & 50 & 80.1117 & 81.0354 & 1.1530 \\
\hline 10 & 45.2514 & 46.4480 & 2.6443 & 60 & 85.4749 & 85.4461 & 0.0337 \\
\hline 12 & 49.6089 & 49.9284 & 0.6440 & 70 & 89.162 & 89.8055 & 0.7217 \\
\hline 15 & 53.6313 & 54.2571 & 1.1669 & 80 & 93.1844 & 93.9422 & 0.8132 \\
\hline 19 & 57.9888 & 58.4119 & 0.7296 & 90 & 97.8771 & 98.1924 & 0.3221 \\
\hline
\end{tabular}

(a)

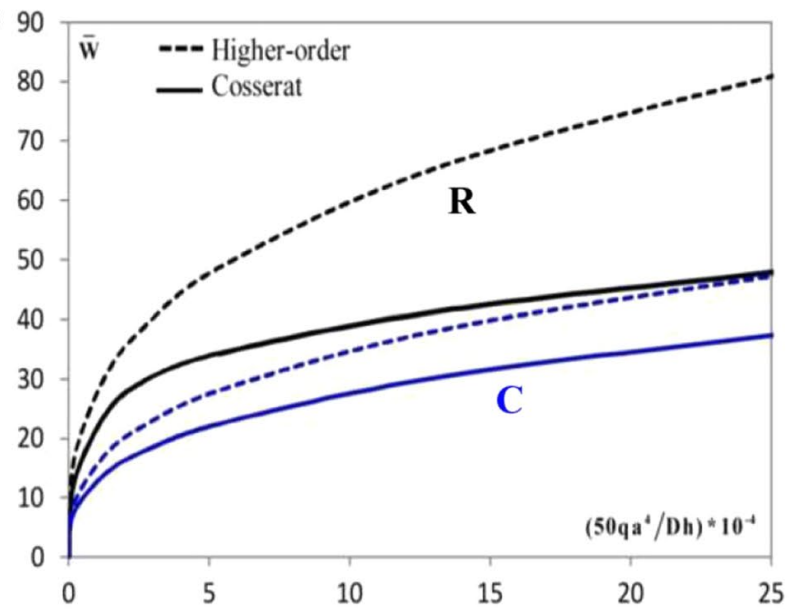

(b) 10

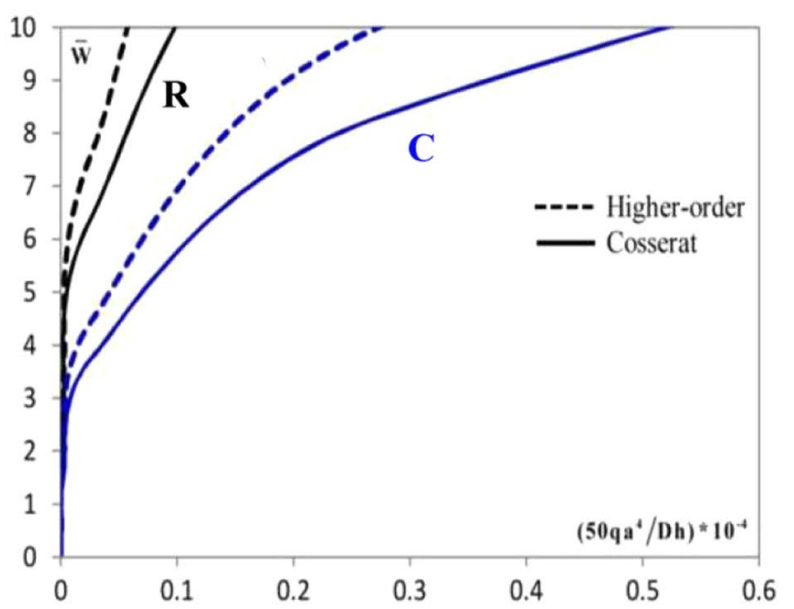

Fig. 7 Comparison of the center deflection for rectangular plate under uniform load: (a) main figure; (b) better view, roller (R), fully clamped (C)

shown in this table, the presented numerical results are very close to exact solution.

Table 2 shows nondimensional stresses in $x_{2}$ direction at $x_{1}=\frac{a}{2}, x_{2}=\frac{b}{2}$. Two aspect ratios of $\frac{a}{b}=2,3$ are tested. As the results shown in Table 2, the stresses are in close agreement with literature.

\section{Validation the numerical results of Cosserat theory}

In this section, results of Cosserat numerical solution are compared for two examples. At the first example, consider a simply supported (SSSS) rectangular plate under uniform distributed load (Fig. 5).

In Table $3, \bar{W}$ is as follows:

$\bar{W}=\frac{1}{h} u_{3}\left(\frac{a}{2}, \frac{b}{2}\right)$.

Cosserat theory gives acceptable results in very large deformation (Cosserat and Cosserat 1909). Table 3 shows

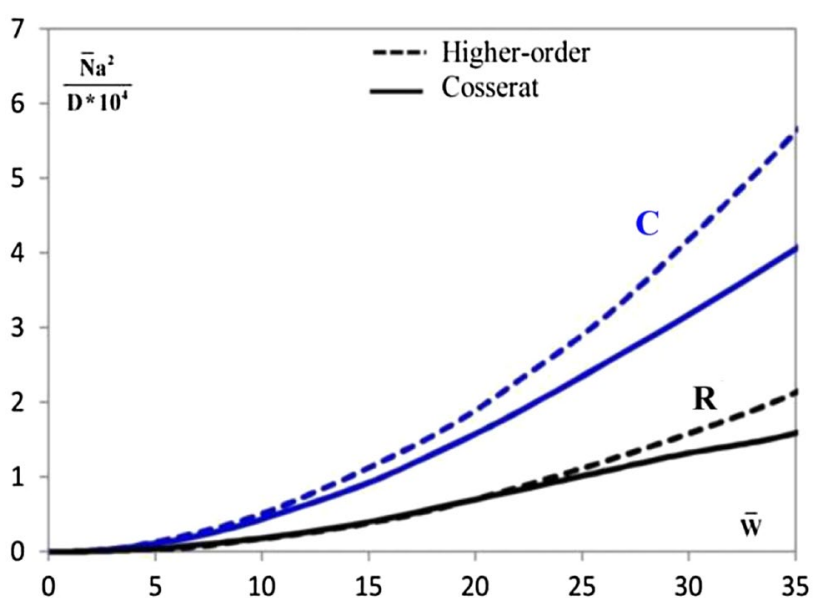

Fig. 8 Comparison of the membrane force at the center of rectangular plate under uniform load, roller $(R)$, fully clamped $(C)$ 
the nondimensional deflection $(\bar{W})$ of the plate under uniform pressure. The pressure was increased to $\bar{W}=49$. According to the revealed results in Table 3, there is a good agreement with the literature.

As the second example, consider a rectangular plate under concentrated load (Fig. 6). Table 4 shows nondimensional deflection at the center of the plate. According to Table 4, by presented numerical solution, deflection of the plate is very close to literature up to very large deformation.

\section{Comparison of Von Karman and Cosserat theories from small to very large deformation}

Nonlinear Von Karman theory results are acceptable for moderately large deformation (Ugural 1981), and noticeably, this theory is not very complex from mathematical point of view. Cosserat theory is a nonlinear advanced theory for simulation of plate behavior in very large deformation, but it is mathematically complicated. In this section, Von Karman and Cosserat theories are compared from small to very large deformation for rectangular and skew plates. This comparison can give acceptable range of Von Karman nonlinear theory for large deformation problems.

\section{Rectangular plate under uniform pressure}

Consider a rectangular plate with $\frac{a}{h}=0.01$. The problem is solved for two boundary conditions: (1) all edges are roller and (2) fully clamped.

Figure 7 shows nondimensional deflection $(\bar{W})$ at the center of the plate using two theories. In addition, a better view of Fig. 7 is drawn. It can be seen from Fig. $7 b$ that two theories are very close to each other until $\bar{W}=3$ for fully clamped boundary condition and $\bar{W}=5$ for roller boundary condition. As the compared results of membrane force shown in Fig. 8, for clamped case, membrane forces until $\frac{\bar{N} a^{2}}{D \times 10^{4}}=1.3$ are close to each other and for simply supported case up to $\frac{\bar{N} a^{2}}{D \times 10^{4}}=1.7$, the results of the two theories are matched. It can be seen from Fig. 8 that membrane forces of the two theories are matched up to a range of large deformation.
Fig. 9 Skew plate under uniform load with simply supported boundary condition
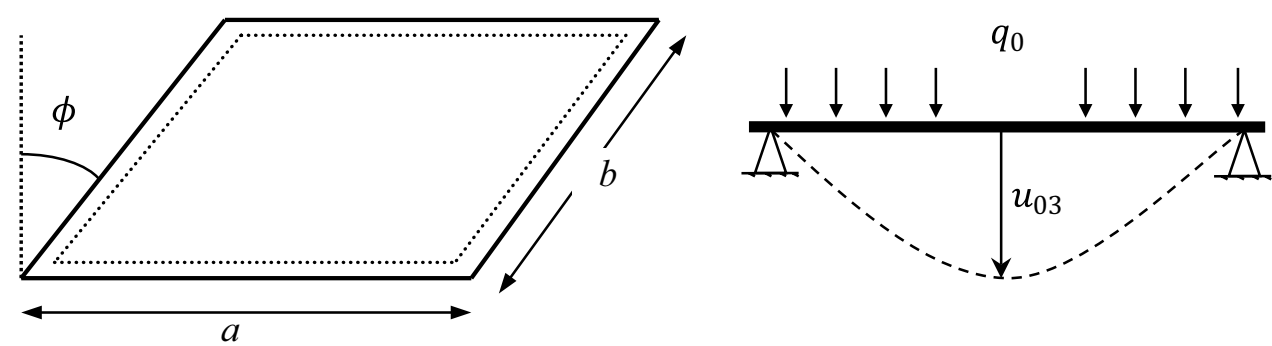

(a) 45

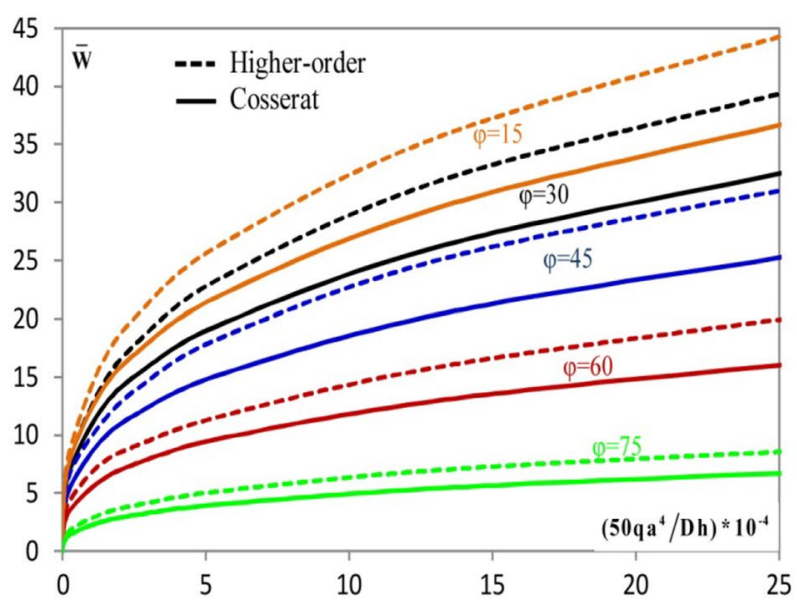

(b) 6

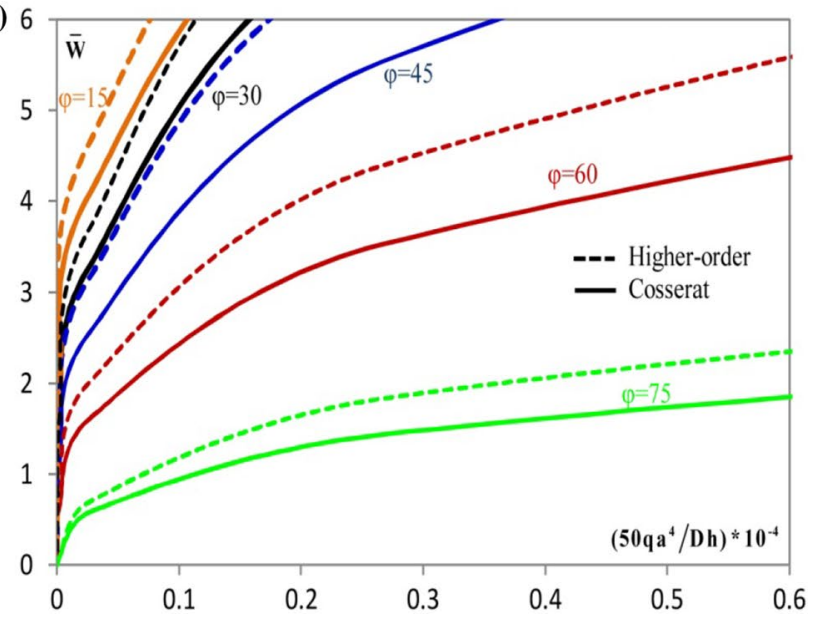

Fig. 10 Comparison of the central deflection of skew plate under uniform load with simply supported boundary condition: (a) main figure; (b) better view 


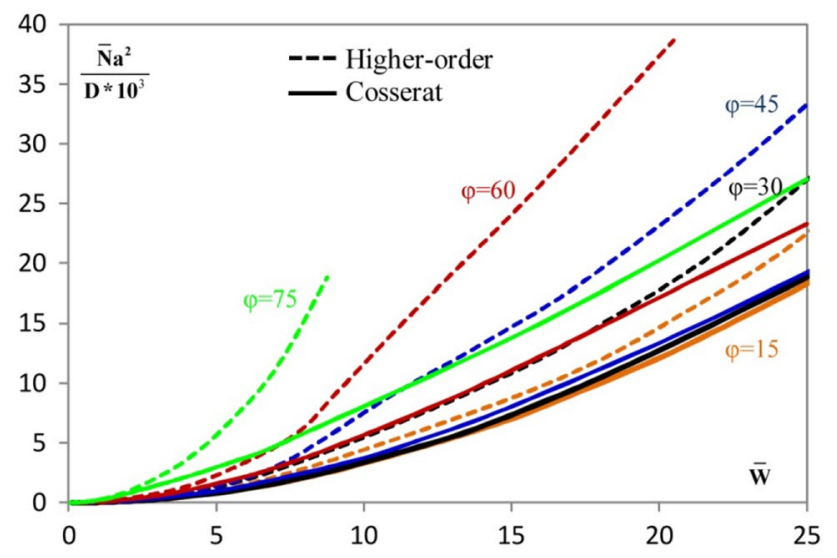

Fig. 11 Comparison of the membrane force of skew plate under uniform load with simply supported boundary condition

\section{Skew plate under uniform pressure}

Consider a skew plate according to Fig. 9. In this section, two theories are compared for five skew plates by $\phi=15^{\circ}, 30^{\circ}, 45^{\circ}, 60^{\circ}, 75^{\circ}$. The plate boundary condition is considered simply supported.

Figure 10 shows nondimensional deflection at the center of the plate versus nondimensional uniform pressure. Numerical solution was applied from small to very large deformation. The results showed that two theories are matched as follows:

For $\phi=15^{\circ}$ up to $\bar{W}=3$

For $\phi=30^{\circ}$ up to $\bar{W}=2.5$

For $\phi=45^{\circ}$ up to $\bar{W}=2$

For $\phi=60^{\circ}$ up to $\bar{W}=1$

For $\phi=75^{\circ}$ up to $\bar{W}=0.5$.

It is obvious that by increasing the skew angle, consistency of two theories decreases. In addition, Fig. 11 indicates nondimensional membrane force in $x_{1}$ direction versus nondimensional deflection at the center of the plate. This figure was drawn up to large deformation. According to Fig. 11, membrane force of two theories is matched as follows:

For $\phi=15^{\circ}$ up to $\frac{\bar{N} a^{2}}{D \times 10^{3}}=0.8$ For $\phi=60^{\circ}$ up to $\frac{\bar{N} a^{2}}{D \times 10^{3}}=0.5$

For $\phi=30^{\circ}$ up to $\frac{\bar{N} a^{2}}{D \times 10^{3}}=0.7 \quad$ For $\phi=75^{\circ}$ up to $\frac{\bar{N} a^{2}}{D \times 10^{3}}=0.3$

For $\phi=45^{\circ}$ up to $\frac{\bar{N} a^{2}}{D \times 10^{3}}=0.6$

\section{Conclusions}

In this study, two theories of HSDT (considering Von Karman) and Cosserat were compared up to large deformation ranges of the skew plates in simply supported and clamped boundary conditions for all edges. Whole domain method was applied for numerical solution. It is notable that according to the obtained results, Von Karman theory was simpler than Cosserat theory in formulation and computation. This comparison suggested acceptable range of Von Karman theory in very large deformation. Moreover, for the rectangular plates, the results obtained by the two theories matched when the maximum nondimensional deflection was approximately $\bar{W}=5$ for simply supported boundary conditions and $\bar{W}=3$ in clamped boundary conditions. Hence, Von Karman theory was valid for deflections greater than thickness up to a specific range for presented boundary condition. In addition, for the skew plates, consistency of two theories depended on skew angle. By increasing the skew angle, consistency of two theories decreases. The results illustrated that for plates with great skew angle, Von Karman theory deviated from Cosserat theory even in deflections smaller than thickness.

Open Access This article is distributed under the terms of the Creative Commons Attribution 4.0 International License (http://creativeco mmons.org/licenses/by/4.0/), which permits unrestricted use, distribution, and reproduction in any medium, provided you give appropriate credit to the original author(s) and the source, provide a link to the Creative Commons license, and indicate if changes were made.

\section{References}

Amiri Rad A, Panahandeh-Shahraki D (2014) Buckling of cracked functionally graded plates under tension. Thin Walled Struct 84:26-33

Cosserat E, Cosserat F (1909) Théorie Des Corps Déformables. Hermann, Paris

Duc ND, Cong PH (2013) Nonlinear postbuckling of symmetric $\mathrm{S}$-FGM plates resting on elastic foundations using higher order shear deformation plate theory in thermal environments. Compos Struct 100:566-574

Duc ND, Tung HV (2011) Mechanical and thermal postbuckling of higher order shear deformable functionally graded plates on elastic foundations. Compos Struct 93(11):2874-2881

Fazzolari FA, Banerjee JR, Boscolo M (2013) Buckling of composite plate assemblies using higher order shear deformation theory-an exact method of solution. Thin Walled Struct 71:18-34

Ghassemi A, Shahidi A, Farzin M (2010) A new element for analyzing large deformation of thin Naghdi shell model. Part 1: Elastic. Appl Math Model 34(12):4267-4277

Ghassemi A, Shahidi A, Farzin M (2011) A new element for analyzing large deformation of thin Naghdi shell model. Part II: Plastic. Appl Math Model 35(6):2650-2668

Kumar JS, Reddy BS, Reddy CE (2011) Nonlinear bending analysis of functionally graded plates using higher order theory. Int J Eng Sci Technol 3(4):3010-3022 
Lee C-Y, Kim J-H (2014) Degradation of thermal postbuckling behaviors of functionally graded material in aero-hygrothermal environments. Compos Struct 118:228-233

Mechab I, Atmane H, Tounsi A, Belhadj H, Bedia E (2010) A two variable refined plate theory for the bending analysis of functionally graded plates. Acta Mech Sin 26(6):941-949

Neff P (2004) A geometrically exact Cosserat shell-model including size effects, avoiding degeneracy in the thin shell limit. Part I: Formal dimensional reduction for elastic plates and existence of minimizers for positive Cosserat couple modulus. Contin Mech Thermodyn 16(6):577-628

Nguyen-Xuan H, Thai CH, Nguyen-Thoi T (2013) Isogeometric finite element analysis of composite sandwich plates using a higher order shear deformation theory. Compos B Eng 55:558-574

Ovesy HR, Taghizadeh M, Kharazi M (2012) Post-buckling analysis of composite plates containing embedded delaminations with arbitrary shape by using higher order shear deformation theory. Compos Struct 94(3):1243-1249

Phung-Van P, Nguyen-Thoi T, Tran LV, Nguyen-Xuan H (2013) A cell-based smoothed discrete shear gap method (CS-DSG3) based on the C0-type higher-order shear deformation theory for static and free vibration analyses of functionally graded plates. Comput Mater Sci 79:857-872

Reddy JN (1984) A simple higher order theory for laminated composite plates. J Appl Mech 51(4):745-752

Sahmani S, Ansari R (2013a) On the free vibration response of functionally graded higher-order shear deformable microplates based on the strain gradient elasticity theory. Compos Struct 95:430-442

Sahmani S, Ansari R (2013b) Size-dependent buckling analysis of functionally graded third-order shear deformable microbeams including thermal environment effect. Appl Math Model 37(23):9499-9515

Sahmani S, Bahrami M, Ansari R (2014) Nonlinear free vibration analysis of functionally graded third-order shear deformable microbeams based on the modified strain gradient elasticity theory. Compos Struct 110:219-230

Shahidi AR, Mahzoon M, Saadatpour MM, Azhari M (2007) Nonlinear static analysis of arbitrary quadrilateral plates in very large deflections. Commun Nonlinear Sci Numer Simul 12(5):832-848

Shariyat M (2010a) A generalized high-order global-local plate theory for nonlinear bending and buckling analyses of imperfect sandwich plates subjected to thermo-mechanical loads. Compos Struct 92(1):130-143

Shariyat M (2010b) Non-linear dynamic thermo-mechanical buckling analysis of the imperfect sandwich plates based on a generalized three-dimensional high-order global-local plate theory. Compos Struct 92(1):72-85
Shi G (2007) A new simple third-order shear deformation theory of plates. Int J Solids Struct 44(13):4399-4417

Simo JC (1993) On a stress resultant geometrically exact shell model. Part VII: shell intersections with 56-DOF finite element formulations. Comput Methods Appl Mech Eng 108(3-4):319-339

Simo JC, Fox DD (1989) On a stress resultant geometrically exact shell model. Part I: formulation and optimal parametrization. Comput Methods Appl Mech Eng 72(3):267-304

Simo JC, Kennedy JG (1992) On a stress resultant geometrically exact shell model. Part V. Nonlinear plasticity: formulation and integration algorithms. Comput Methods Appl Mech Eng 96(2):133-171

Simo JC, Fox DD, Rifai MS (1989) On a stress resultant geometrically exact shell model. Part II: the linear theory; computational aspects. Comput Methods Appl Mech Eng 73(1):53-92

Simo JC, Fox DD, Rifai MS (1990a) On a stress resultant geometrically exact shell model. Part III: computational aspects of the nonlinear theory. Comput Methods Appl Mech Eng 79(1):21-70

Simo JC, Rifai MS, Fox DD (1990b) On a stress resultant geometrically exact shell model. Part IV: variable thickness shells with through-the-thickness stretching. Comput Methods Appl Mech Eng 81(1):91-126

Swaminathan K, Naveenkumar DT (2014) Higher order refined computational models for the stability analysis of FGM plates-analytical solutions. Eur J Mech A Solids 47:349-361

Tahouneh V (2016) Using an equivalent continuum model for 3D dynamic analysis of nanocomposite plates. Steel Compos Struct 20(3):623-649

Tahouneh V, Naei MH (2016a) The effect of multi-directional nanocomposite materials on the vibrational response of thick shell panels with finite length and rested on two-parameter elastic foundations. Int J Adv Struct Eng 8(1):11-28

Tahouneh V, Naei MH (2016b) Free vibration and vibrational displacements analysis of thick elastically supported laminated curved panels with power-law distribution functionally graded layers and finite length via 2D GDQ method. J Sandw Struct Mater 18(3):263-293

Ugural AC (1981) Stresses in plates and shells. McGraw-Hill, New York

Yang J, Liew KM, Kitipornchai S (2006) Imperfection sensitivity of the post-buckling behavior of higher-order shear deformable functionally graded plates. Int J Solids Struct 43(17):5247-5266

Publisher's Note Springer Nature remains neutral with regard to urisdictional claims in published maps and institutional affiliations. 